\title{
Benchmark Rovibrational Linelists and Einstein A-coefficients for the Primordial Molecules and Isotopologues
}

\author{
Paulo H. R. Amaral ${ }^{1}$ (10), Leonardo G. Diniz ${ }^{2}$ (1) Keith A. Jones $^{3}$, Monika Stanke ${ }^{4}$, Alexander Alijah ${ }^{5}$ (1), \\ Ludwik Adamowicz ${ }^{3,6}$ (i), and José R. Mohallem ${ }^{1}$ (1) \\ ${ }^{1}$ Laboratório de Átomos e Moléculas Especiais, Departamento de Física, ICEx, Universidade Federal de Minas Gerais, P. O. Box 702, $30123-970$ Belo Horizonte, \\ MG, Brazil; phramaral@gmail.com \\ ${ }^{2}$ Coordenação de Ciências, Centro Federal de Educação Tecnológica de Minas Gerais, 30.421-169, Belo Horizonte, MG, Brasil; leogabriel@ cefetmg.br \\ ${ }^{3}$ Department of Chemistry and Biochemistry, The University of Arizona, Tucson, AZ 85721, USA; joneska@email.arizona.edu \\ ${ }^{4}$ Institute of Physics, Faculty of Physics, Astronomy, and Informatics, Nicolaus Copernicus University, ul. Grudziądzka 5, Toruń, PL 87-100, Poland \\ monika@ fizyka.umk.pl \\ ${ }^{5}$ Groupe de Spectrométrie Moléculaire et Atmosphérique, GSMA, UMR CNRS 7331, Université de Reims Champagne-Ardenne, U.F.R. Sciences Exactes et \\ Naturelles, Moulin de la Housse B.P. 1039, F-51687 Reims Cedex 2, France; alexander.alijah@univ-reims.fr \\ ${ }^{6}$ Interdisciplinary Center for Modern Technologies, Nicolaus Copernicus University, ul. Wileńska 4, Toruń, PL 87-100, Poland; ludwik@email.arizona.edu, \\ rachid@ fisica.ufmg.br \\ Received 2019 January 11; revised 2019 April 30; accepted 2019 May 2; published 2019 June 18
}

\begin{abstract}
Complete benchmark rovibrational energy linelists calculated for the primordial polar molecules of the universe, namely $\mathrm{HD}^{+}, \mathrm{HD}$, and the $\mathrm{HeH}^{+}$isotopologues, with accuracy up to $10^{-2} \mathrm{~cm}^{-1}$ for low-lying states, are presented. To allow for these calculations to be performed, new high-accuracy potential energy curves, which include the diagonal Born-Oppenheimer adiabatic corrections and the leading relativistic corrections, are determined. Also, a new approach for calculating non-adiabatic corrections involving an effective vibrational nuclear mass obtained based on the atoms-in-molecules theory is employed. The vibrational and rotational masses are taken as being different and dependent on the nuclear distance. Accurate dipole moment curves are calculated and used to generate lists of Einstein A-coefficients. The energy linelists and the sets of Einstein A-coefficients for HD are upgrades of previous calculations including quasibound states, while for $\mathrm{HD}^{+}$and $\mathrm{HeH}^{+}$and its isotopologues the present results represent significant improvement over the previous calculations. The results obtained here suggest that, with the inclusion of the non-adiabatic corrections, the accuracy limit at least for low-lying states might have been reached. Thus, further progress should involve accounting for even smaller effects such as the quantumelectrodynamics corrections. The present results represent the state-of-the-art of theoretical spectroscopy of the primordial polar molecules.
\end{abstract}

Key words: astrochemistry - molecular data - molecular processes

Supporting material: tar.gz file

\section{Introduction}

Two main factors, abundance and polarity, compete in the discussion of the spectroscopic role played by the smallest primordial molecules of the universe. This covers the period that started from their genesis when the temperature reached less than $4000 \mathrm{~K}$ (the recombination era) to the star formation at later times (Lepp et al. 2002). The homonuclear molecules $\mathrm{H}_{2}$ and $\mathrm{H}_{2}^{+}$are expected to be the more abundant ones, but their spectroscopic contributions are negligible because they originate from quadrupole electric and/or dipole magnetic transitions. The ionic polar $\mathrm{HD}^{+}$and $\mathrm{HeH}^{+}$and its isotopologues, on the other hand, are expected to be less abundant but their spectroscopic contribution is much larger due to their much more intense electric dipole transitions. The slightly polar HD lies in terms of the spectroscopic contribution in between the above two groups of molecules due to its larger abundance (Galli \& Palla 1998), but a small (though finite) spectroscopic contribution originating from its very small electric dipole moments. The $\mathrm{HD}$ and $\mathrm{HeH}^{+}$molecules play important roles in the study of interstellar matter and star- and planet-forming regions. Outside the scope of astrophysics, accurate calculations for $\mathrm{HD}^{+}$are of interest for metrology, in connection with the development of molecular clocks, as well as for the physics of fundamental constants, namely the improvement of the electron/proton mass ratio (Patra et al. 2018). Notably, $\mathrm{HeH}^{+}$ has been detected very recently in space (Güsten et al. 2019).

But, in order to deepen the understanding of the cooling processes in the primordial universe and to study many other processes that became important in the later stages of the evolution of the universe, rovibrational linelists, as well as lists of Einstein A-coefficients, for these molecules, need to be determined with high accuracy. The more recent works that reported the data considered in the present work, namely energy linelists and Einstein A-coefficients, are Coppola et al. (2011) for $\mathrm{HD}^{+}$, Pachucki \& Komasa (2010) for HD, and Engel et al. (2005) for $\mathrm{HeH}^{+}$and its isotopologues. In Tung et al. (2012) some accurate transitions are reported for $\mathrm{HeH}^{+}$ and its isotopologues. Pachucki \& Komasa (2012) reported dissociation energies of the lowest rovibrational levels $(v \leqslant 5$ and $J \leqslant 14$ ) only for ${ }^{4} \mathrm{HeH}^{+}$. The corresponding total energy values taken from Coppola et al. (2011) and Engel et al. (2005) are tabulated in the website Exomol (Tennyson et al. 2016). Exomol databases for $\mathrm{HD}^{+}$and $\mathrm{HeH}^{+}$and isotopologues are mostly out-of-date. Pachuki and Komasa's databases for HD are very accurate but lack quasibound states, while their databases for ${ }^{4} \mathrm{HeH}^{+}$(without other isotopologues) do not display all transitions and lack quasibound states and A-coefficients. Thus, the present work involving generation of state-of-the-art linelists, as well as complete sets of Einstein 
A-coefficients for the key primordial systems, is very much in order.

Only a few groups have capabilities today to produce very accurate potential energy curves (PECs) for the electronic ground states of the molecules considered in the present work that include relativistic and diagonal Born-Oppenheimer (DBOC) corrections. In order to achieve an accuracy of at least $10^{-1} \mathrm{~cm}^{-1}$, the inclusion of non-adiabatic corrections to the energy levels is imperative.

Non-adiabatic corrections are more difficult to obtain from $\mathrm{ab}$ initio calculations. One way to include these corrections is by using effective masses of the nuclei in determining rovibrational transitions. The effective masses simulate the electron dragging by the nuclei when the molecule is vibrating and/or rotating (Kutzelnigg 2007; Mohallem et al. 2011). The simple use of constant nuclear masses for the rotation calculations and atomic (or dissociation) masses for the vibration calculations already significantly improves the results. However, to reach the $10^{-1} \mathrm{~cm}^{-1}$ accuracy in the calculations of the energy levels more sophisticated effectivemass models need to be employed. In these models, the effective vibrational and rotational masses need to depend on the inter-nuclear distance (i.e., the masses should be $R$-dependent).

In one of our previous papers (Diniz et al. 2018), the most accurate linelists and radiative cooling functions for the $\mathrm{LiH}$ isotopologues were calculated. In the calculations very accurate PEC and dipole moment curves (DMC) reported before (Diniz et al. 2016) were employed. The non-adiabatic corrections were obtained with the use of a constant reduced nuclear mass for rotations and a $R$-dependent mass taken from Diniz et al. (2015; obtained in valence-bond calculations) for vibrations.

In the present work, further progress in calculating PECs (Jones et al. 2016a), as well as a new procedure for calculating non-adiabatic corrections, are reported. The approach is used in state-of-the-art rovibrational calculations for the target molecules. The progress in the PEC calculations includes implementation of improved procedures for calculating the leading relativistic corrections. The progress in calculating the nonadiabatic effects includes implementation of a procedure based the atoms-in-molecules (AIM) core-valence partition of the electronic density (Amaral \& Mohallem 2017). The partition allows for the determination of the fractions of the electron density that needs to be added to the nuclear masses to produce the effective vibrational reduced masses. Both implementations have allowed us to achieve the $10^{-1} \mathrm{~cm}^{-1}$ accuracy in the transition energy calculations.

The present paper is organized as follows. Section 2 contains a brief discussion of the basic spectroscopy equations for calculating the rovibrational energy levels and A-coefficients. Section 3 describes the methodology used to calculate the PECs and DMCs. In Section 4 the AIM approach for calculating effective nuclear masses is discussed. In Section 5 the results for which comparison with available experimental and theoretical data is possible are presented. The complete set of the data calculated in this work are presented in the tar.gz package in the Appendix. The paper is concluded with a general discussion and a summary.

\section{Basic Equations}

For an electronic state well separate from other states (the socalled one-state approximation), Schrödinger's radial equation for a diatomic molecule is (Bunker \& Moss 1977):

$$
\left[-\frac{1}{2 \mu_{\mathrm{vib}}} \frac{d^{2}}{d R^{2}}+E_{e}(R)+\frac{J(J+1)}{2 \mu_{\mathrm{rot}} R^{2}}-E_{v J}\right] \chi_{v J}(R)=0,
$$

where $\mu_{\text {vib }}$ and $\mu_{\text {rot }}$ are the vibrational and rotational reduced masses, respectively, $E_{e}(R)$ is the PEC, and $E_{v J}$ and $\chi_{v J}$ are the eigenvalues and rovibrational wavefunctions of the quantum state characterized by the vibrational and rotational quantum numbers $v$ and $J$. In the electronic calculations, in which the PEC is obtained, the nuclei are considered to have infinite masses (the Born-Oppenheimer (BO) approximation). To solve the above radial equation, Equation (1), the masses $\mu_{\mathrm{vib}}$ and $\mu_{\text {rot }}$ are usually assumed to be the same and equal to the reduced mass of the nuclei. This assumption is not used in the present work because, as shown later in this work, the $R$-dependence of the masses is found to be crucial to improve the calculation accuracy. In highly accurate calculations, diagonal Born-Oppenheimer and relativistic corrections are typically added to the BO PEC before solving Equation (1). Once this equation is solved and a set of rovibrational wavefunctions and the corresponding energies is obtained, the Einstein A-coefficients (or dipole transition probabilities) are calculated using the DMC, $d(R)$, as

$$
A_{v^{\prime} J^{\prime} v^{\prime \prime} J^{\prime \prime}}=\left(\frac{16 \pi^{3}}{3 \epsilon_{0} h}\right) \frac{S\left(J^{\prime}, J^{\prime \prime}\right)}{2 J^{\prime}+1} \nu^{3}\left|\left\langle\chi_{v^{\prime} J^{\prime}}|d(R)| \chi_{v^{\prime \prime} J^{\prime \prime}}\right\rangle\right|^{2},
$$

where $S\left(J^{\prime}, J^{\prime \prime}\right)$ are the Hönl-London rotational intensity factors, $\epsilon_{0}$ is the permittivity of vacuum, $h$ is the Plank constant, and $\nu$ is the transition frequency. Here, the symbols $\left(v^{\prime}, J^{\prime}\right)$ and $\left(v^{\prime \prime}, J^{\prime \prime}\right)$ denote, respectively, the upper and lower rovibrational states between which a spontaneous transition occurs. For further details of the theoretical procedure the reader is referred to Diniz et al. (2016).

\section{Potential Energy and DMCs}

The present-day experimental high-resolution spectroscopy of small molecules has an accuracy of less than $10^{-2} \mathrm{~cm}^{-1}$ (see, for example, Sprecher et al. 2010). Reaching similar accuracy in theoretical calculations is a serious challenge for theoreticians. The starting point for rovibrational calculations are usually highly accurate PECs obtained within the BornOppenheimer approximation. At this level, the accuracy of state-of-the-art curves is better that $0.002 \mathrm{~cm}^{-1}$ for each BO point (Tung et al. 2012), which results, without further corrections, in an accuracy of at most tenths $\mathrm{of} \mathrm{cm}^{-1}$ for the rovibrational energy levels. The adiabatic correction (DBOC) amounts to about $10^{2} \mathrm{~cm}^{-1}$ and contributes within $10^{0}-10^{1} \mathrm{~cm}^{-1}$ to the energy levels. Thus they must be included in the PECs. As demonstrated by Tung et al. (2012), these terms can be presently evaluated with an accuracy better than $0.0005 \mathrm{~cm}^{-1}$. The leading relativistic corrections for two electron systems (Kediziera et al. 2006) are about one order of magnitude smaller than the adiabatic correction, but when summed with the leading quantum-electrodynamics (QED) corrections they become more relevant with increasing vibrational quantum number $v$. The remaining differences between the calculated and the experimental levels are mainly due to non-adiabatic corrections. 
The calculations performed in the present work are based on the new high accurate PECs presented in the tar.gz package Appendix. They were constructed by the variational minimization of the expectation value of the $\mathrm{BO}$ electronic Hamiltonian for each point of the PEC, as done in Tung et al. (2012). The electronic wavefunctions are expanded on a basis of explicitly correlated Gaussian functions (ECGs) with shifted centers. The functional form of the basis functions can be seen in Equation (2) in Pavanello et al. (2008). The optimization of the nonlinear parameters of the ECGs is carried out in a cyclic process as described in Jones et al. (2016b). This cyclic optimization of individual functions was shown to be effective for an allparticle ECG basis in non-Born-Oppenheimer calculations of diatomic molecules (Jones et al. 2016b), and proved effective in this work as well. The analytic expression of the gradient of the energy with respect to the nonlinear ECG parameters is employed to speed up the optimization (Pavanello et al. 2008). In the process of growing the basis set new functions are added to the basis as described in Jones et al. (2016a). This addition involves identifying a subset of the functions that have the greatest energy contribution. Next, these functions, called candidate functions, have their nonlinear parameters perturbed slightly, followed by optimization of their nonlinear parameters. After checking the optimized functions for linear dependence with themselves and with the functions already included in the basis set and eliminating the functions that cause the linear dependence, the new most contributing optimized candidate functions are included in the basis set.

In order to construct a PEC, a single point on the curve is first optimized. Usually this point corresponds to the equilibrium inter-nuclear distance. A new point in proximity to the optimized point is then selected and a procedure based on the Gaussian product theorem is used to shift the centers of the ECGs optimized for the first PEC point to the new point (Cencek \& Kutzelnigg 1997). The nonlinear parameters of the ECGs of the new point are then re-optimized in the way previously mentioned. After the optimization of the ECG basis set is completed and the corresponding electronic energy and the wavefunction is calculated, the next PEC point is selected and its ECG basis set is generated and optimized. This is again followed by the energy and wavefunction calculations. The procedure continues until the whole PEC is calculated. The density of the points along the PEC is uneven-more dense in the range where PEC changes more and less dense for more linearly behaving parts of the PEC.

The calculations of the DBOCs for the various isotopologues are carried out as described in Tung et al. (2012) and Cencek \& Kutzelnigg (1997). As pointed out, the accuracy of the calculations is about $0.0005 \mathrm{~cm}^{-1}$. The DBOCs are added to the PEC to generate PECs for different isotopologues.

The main improvement in the PECs in this work comes from including the leading relativistic corrections calculated with the newly derived algorithms involving expectation values of the operators representing the corrections in the electronic relativistic Breit-Pauli Hamiltonian (Bethe \& Salpeter 1977). The relativistic corrections to the electronic energy include mass-velocity, one- and two-electron Darwin, orbit-orbit, and spin-spin corrections. The expectation values are calculated using the ECGs optimized for each PEC point. The details of the derivation and implementation of the algorithms are outlined in Stanke et al. (2016a, 2016b).
The $\mathrm{HeH}^{+}$DMC needed for the evaluation of the Einstein A-coefficients are generated in the same calculations where the energies and wavefunctions for the PEC points are determined. The DMC is the same for ${ }^{4} \mathrm{HeH}^{+}$and its isotopologues. Details on the procedure followed here can be found in Diniz et al. (2016).

Computations of the isotopic dipole moments of $\mathrm{HD}^{+}$and HD demand special treatment. The DMC for $\mathrm{HD}^{+}$is also obtained here, by moving the center of coordinates to the center of mass of the nuclei. On the other hand, the DMC for the neutral HD is not performed here. Instead, it is taken from Pachucki \& Komasa (2008), and is the same DMC used by Coppola et al. (2011). The new PECs and DMCs obtained for the particular grids of $R$ used for the considered systems, are shown in the tar.gz package.

\section{Non-adiabatic Effects on the Energy Levels}

Along with the high accuracy of the PEC calculations, accounting for the non-adiabatic effects is crucial to improve the energy linelists. In cases of well-isolated ground electronic states weakly coupled to excited states, non-adiabatic corrections to the vibrational energy levels have their origin in the dragging of some fraction of the electron density by the nuclei when they rotate and vibrate (this effect contributes to the inaccuracy of the adiabatic model). Considering the physical nature of the non-adiabatic effect, it has been accounted for with increasing accuracy by taking the difference between the rovibrational energies calculated with nuclear masses and those obtained with empirical effective masses (Kutzelnigg 2007; Mohallem et al. 2011; Diniz et al. 2012) (the calculations with different masses are performed with the nuclear equation, Equation (1)). However, the empirical mass models introduced so far have been unable to account for the whole non-adiabatic effect.

In our recent paper, using the AIM theory developed within the iterative stockholder framework of Hirshfeld (1977) (SAIM), a core-valence electron separation (CVSAIM) model was introduced (Amaral \& Mohallem 2017). The CVSAIM core electron densities are considered as moving along with the corresponding nuclei as these nuclei vibrate and rotate. These densities are $R$-dependent and should be added to the nuclear masses to determine the effective nuclear masses. These effective masses are to be used in the rovibrational nuclear equation to account for the non-adiabatic effects in the calculations of the rovibrational energy levels. As shown in Amaral \& Mohallem (2017), this accounting for the nonadiabatic effects is so far the most accurate for the systems studied in that work. In the procedure there is no need to use any empirical scaling of the correction curves, such as, for example, the scaling used in Diniz et al. (2012).

The CVSAIM method for attributing effective masses to the nuclei can be outlined as follows. In the first step of the approach the one-electron density of the system, $\rho_{\text {mol }}(r)$, is calculated for all PEC points. In the next step the CVSAIM density partition is performed for the density at each of the PEC points. The CVSAIM method is based on the AIM concept and the SAIM scheme introduce by Hirshfeld (1977). For example, let us take a diatomic molecule, $\mathrm{AB}$, and let $\rho_{\mathrm{A}}^{0}$ and $\rho_{\mathrm{B}}^{0}$ be the densities of the isolated atoms. Let us call the sum of the two atomic densities, $\rho_{\mathrm{mol}}^{0}=\rho_{\mathrm{A}}^{0}+\rho_{\mathrm{B}}^{0}$, the promolecule density. Then, in the first-order approximation, the density of SAIM A, 
$\rho_{\mathrm{A}}^{1}$, is determined as

$$
\rho_{\mathrm{A}}^{1}=\frac{\rho_{\mathrm{A}}^{0}}{\rho_{\mathrm{mol}}^{0}} \rho_{\mathrm{mol}},
$$

with an analogous definition for $\rho_{\mathrm{B}}^{1}$. The same procedure is followed for the $n$ th-order approximation, namely,

$$
\rho_{\mathrm{A}}^{n}=\frac{\rho_{\mathrm{A}}^{n-1}}{\rho_{\mathrm{mol}}^{n-1}} \rho_{\mathrm{mol}} .
$$

The iterative procedure called ISA (iterated stockholder atoms, Lillestonen \& Wheatley 2008), which is explained in Amaral \& Mohallem (2017), allows determination of the SAIM densities of atoms $\mathrm{A}$ and $\mathrm{B}, \rho_{\mathrm{A}}$ and $\rho_{\mathrm{B}}$. The sum of these densities is the molecular density

$$
\rho_{\mathrm{mol}}=\rho_{\mathrm{A}}+\rho_{\mathrm{B}}
$$

SAIM introduces the concept of imitated atoms in the molecule, while CVSAIM introduces the idea that each SAIM has two different $R$-dependent parts, a core part that moves along with the nucleus when it vibrates and a valence part that does not participate in this motion. For the purpose of the present application of the SAIM approach, the core fraction of the CVSAIM for each atom must be identified. With this in mind, an effective one-electron potential for each AIM is constructed from the molecular density using the following procedure.

For each atomic center, say, atomic center A, its charge, $q\left(r_{\mathrm{A}}\right)$, is obtained by numerical integration:

$$
q\left(r_{\mathrm{A}}\right)=\int \rho_{\mathrm{mol}} d \tau,
$$

where the integration is performed over a sphere of volume $\tau$ in which $r$ varies from 0 to $r_{\mathrm{A}}$. The effective potential, $V_{\mathrm{A}}\left(r_{\mathrm{A}}\right)$, is

$$
V_{\mathrm{A}}\left(r_{\mathrm{A}}\right)=-\frac{Z_{\mathrm{A}}+q\left(r_{\mathrm{A}}\right)}{r_{\mathrm{A}}}, \quad q\left(r_{\mathrm{A}}\right)<0,
$$

where $Z_{\mathrm{A}}$ is the charge of nucleus $\mathrm{A}$, and $r_{\mathrm{A}}$ ranges from 0 to the value that is determined from the condition: $V_{\mathrm{A}}\left(r_{\mathrm{A}}\right)=0$. The same procedure is followed for atom B.

Once the molecular density is partitioned into the CVSAIM components, the core fraction of the electron density of CVSAIM A, $\rho_{\mathrm{A}, c}$, is chosen as the part of the whole SAIM density preponderantly subject to the potential $V_{\mathrm{A}}$, that is, corresponding to the region where $V_{\mathrm{A}}$ is more attractive than $V_{\mathrm{B}}$ (Figure 4 of Amaral \& Mohallem (2017) illustrates this feature). The same procedure is followed to obtain the core fraction of the CVSAIM density of atom B, $\rho_{\mathrm{B}, c}$. It should be noted that for homonuclear molecules the cores are easily identified as the non-superposed parts of the CVSAIM densities. Then, the superposing part of the densities correspond to the stationary density of the valence electrons (see Figure 1 in Amaral \& Mohallem 2017).

The electronic masses to be added to the nuclear masses, in order to determine the effective nuclear masses, are obtained by numerical integration of the core part of the CVSAIM density for each atomic center. With that the effective mass of, for example, nucleus $\mathrm{A}$, is:

$$
m_{\mathrm{A}, \mathrm{vib}}(R)=m_{\mathrm{A}}+m_{e} \int \rho_{\mathrm{A}, c}(r, R) d \tau,
$$

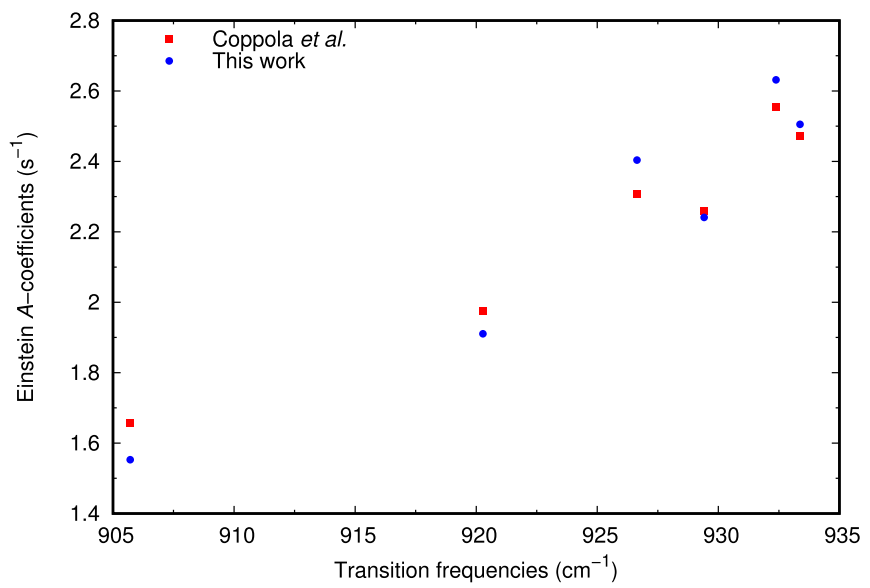

Figure 1. Comparison of the Einstein A-coefficients for the (18-16) band of the $R$-branch of $\mathrm{HD}^{+}$

where $m_{\mathrm{A}}$ is the mass of the bare nucleus $\mathrm{A}$ and $m_{e}$ is the electron mass, with an analogous expression for $m_{\mathrm{B}, \mathrm{vib}}(R)$. Once $m_{\mathrm{A} \text {,vib }}(R)$ and $m_{\mathrm{B}, \mathrm{vib}}(R)$ are determined for all grid points of the PEC, the effective vibrational reduced mass is calculated as

$$
\mu_{\mathrm{vib}}(R)=\frac{m_{\mathrm{A}, \mathrm{vib}}(R) m_{\mathrm{B}, \mathrm{vib}}(R)}{m_{\mathrm{A}, \mathrm{vib}}(R)+m_{\mathrm{B}, \mathrm{vib}}(R)} .
$$

Vibrational energies that include the non-adiabatic corrections are obtained by solving the vibrational equation with the effective reduced mass of Equation (9). Either an algorithm that allows for the reduced mass to be $R$-dependent (Alijah \& Duxbury 1990), or an algorithm that employs a constant reduced mass can be used, provided that, in the latter case, the masses are averaged over the vibrational states as

$$
m_{A, v}=\int m_{\mathrm{A}}(R) \chi_{v}(R)^{2} d R,
$$

as described in Mohallem et al. (2011), where $\chi_{v}(R)$ is the wavefunction for vibrational level $v$.

In order to obtain good-quality effective masses using the above-described approach, electronic densities are obtained using the GAMESS program package (Schmidt et al. 1993). The calculations are performed at the full-CI level with the ccpV5Z basis set for HD and $\mathrm{HD}^{+}$and with the cc-pVQZ basis set for $\mathrm{HeH}^{+}$. The inclusion of finite nuclear mass effects (Gonçalves \& Mohallem 2004) in the calculations of the electronic energies and the corresponding wavefunctions, and the subsequent calculations of the CVSAIM from the electronic density, is not needed because the densities obtained from the BO calculations are not significantly different (Mohallem et al. 2011; Diniz et al. 2012).

The use of effective rotational masses is less important in the calculations of the rovibrational levels than the use of effective vibrational masses. So far, there has not been a physically motivated model for determining effective rotational masses such as the models used for determining effective vibrational masses. As the potential in rovibrational equation depends on the quantum number $J$, for a fixed $J$ a model for determining a common vibrational and rotational reduced mass can be established. Also, an empirical approach to determine such a reduced mass can, perhaps, be used. The approach chosen here involves determining an effective reduced rotational mass 
through an interpolation of $R$-dependent reduced mass, which at the limit of small $R$ can be accurately approximated using the nuclear masses and at large $R$ can be approximated using the atomic masses (Diniz et al. 2012). The reasoning behind such an approach is that, for low values of $J$, a good approximation is to use a constant rotational reduced mass equal to $\mu_{\text {rot }}(R)=m_{\mathrm{A}} m_{\mathrm{B}} /\left(m_{\mathrm{A}}+m_{\mathrm{B}}\right)$, since the length of the bond is affected little by the rotation. However, as the values of $J$ become larger and the molecule rotates more vigorously, the bond stretches (to eventually break at dissociation) and the nuclear rotational reduced mass approaches the atomic (or dissociation) reduced mass. Thus, a parameterization of the rotational reduced mass can be considered that, near the equilibrium distance, becomes equal to the reduced mass obtained from the nuclear masses and near dissociation becomes equal to the reduced mass obtained from the atomic masses. Such a behavior of $m_{\mathrm{A} \text {,rot }}$ can be represented by the following formula:

$$
m_{\mathrm{A}, \text { rot }}(R)=m_{\mathrm{A}}+a\left[1-\left[1+e^{\alpha\left(R-R_{T}\right)}\right]^{-1}\right] .
$$

Here, $a$ is the net number of electrons in each atomic fragment (for example, $a=1$ for $\mathrm{H}_{2}, a=\frac{1}{2}$ for $\mathrm{H}_{2}^{+}$), while $\alpha$ and $R_{T}$, the turning-point parameters of the function, are empirical parameters. They are obtained for each system using the procedure described in Diniz et al. (2012). The effective, $R$-dependent rotational reduced mass in Equation (1) is then obtained using Equation (9) with the rotational masses of the nuclei determined as described above.

\section{Transition Energies and Einstein A-coefficients}

In this section, tables of the calculated transition energies for which experimental and previous theoretical data are available and tables of selected Einstein A-coefficients are presented. The complete linelists and Einstein A-coefficients data are shown in the tar.gz package. In the Appendix, extracts from the full material are presented for the ${ }^{3} \mathrm{HeH}^{+}$.

The rovibrational bound states are computed with the renormalized Numerov code that employs coordinate-dependent reduced masses (Alijah \& Duxbury 1990). The numerical integration is performed within the $R$ ranges and for the same $R$ grids used in the PEC calculations. The parameters of the procedure are adjusted to achieve numerical convergence of $10^{-14} E_{h}$.

The energies of resonant ( $Q$, quasibound) states are calculated with the LEVEL16 package (LeRoy 2017) modified to allow the use of different vibrational and rotational masses. As this code does not handle $R$-dependent masses, the effective vibrational and rotational masses are averaged, using Equation (10), over the wavefunctions obtained using nuclear masses in Equation (1).

\section{1. $H D^{+}$and $H D$}

In the cooled primordial plasma and star environments rich in $\mathrm{D}$ atoms, $\mathrm{HD}^{+}$and $\mathrm{HD}$ could be formed by simple chemical reactions and ionization processes mostly discussed in Lepp et al. (2002), where their abundance at large redshifts are also discussed.

The generation of linelists for these species (polar and apolar) and their isotopologues is thus of great relevance to the study of the primordial plasma. Experimental energies for some transitions of the species can be obtained from Carrington et al. (1983)
Table 1

$\mathrm{HD}^{+}$- Comparison between Calculated Vibrational Term Values and the Quasi-exact Ones Taken from Balint-Kurti et al. (1990)

\begin{tabular}{|c|c|c|c|}
\hline$v \rightarrow 0$ & Quasi Exact $^{\mathrm{a}}$ & Coppola $^{\mathrm{b}}$ & This Work \\
\hline 1 & 1912.9954 & -1.1002 & -0.0194 \\
\hline 2 & 3729.8563 & -1.5577 & -0.0342 \\
\hline 3 & 5453.4437 & -0.7228 & -0.0434 \\
\hline 4 & 7086.2436 & 2.1810 & -0.0530 \\
\hline 5 & 8630.3773 & 7.7749 & -0.0598 \\
\hline 6 & 10087.6081 & 16.2648 & -0.0602 \\
\hline 7 & 11459.3429 & 27.3092 & -0.0567 \\
\hline 8 & 12746.6309 & 40.0948 & -0.0469 \\
\hline 9 & 13950.1552 & 53.5150 & -0.0316 \\
\hline 10 & 15070.2225 & 66.3806 & -0.0145 \\
\hline 11 & 16106.7442 & 77.5729 & 0.0117 \\
\hline 12 & 17059.2135 & 86.1407 & 0.0360 \\
\hline 13 & 17926.6727 & 91.3406 & 0.0656 \\
\hline 14 & 18707.6747 & 92.6380 & 0.1124 \\
\hline 15 & 19400.2309 & 89.6969 & 0.1655 \\
\hline 16 & 20001.7545 & 82.3592 & 0.2218 \\
\hline 17 & 20508.9903 & 70.6362 & 0.2841 \\
\hline 18 & 20917.9639 & 54.7035 & 0.3717 \\
\hline 19 & 21223.9914 & 34.9411 & 0.5376 \\
\hline 20 & 21422.0170 & 11.7839 & 1.1761 \\
\hline 21 & 21505.8596 & -11.8565 & 8.2245 \\
\hline $\mathrm{rms}$ & & 55.4767 & 1.8211 \\
\hline $\mathrm{rms}^{*}$ & & 59.478 & 0.107 \\
\hline
\end{tabular}

Notes. Coppola: calculated term values from Tennyson et al. (2016). This work: term values calculated in this work. The two last columns contain the differences between calculated and quasi-exact term values. All data are in wavenumbers, $\mathrm{cm}^{-1}$. $\mathrm{rms}^{*}$ discards states with $v$ larger or equal to 18 , for which the average $R$ approaches/exceeds the avoided-crossing region.

a Balint-Kurti et al. (1990).

b Coppola et al. (2011).

(for $\mathrm{HD}^{+}$) and Chuang \& Zare (1987) (for HD). On the theoretical side, while for the one-electron ion $\mathrm{HD}^{+}$quasi-exact values exist for some levels (Wolniewicz \& Poll 1986; BalintKurti et al. 1990; Korobov 2006, 2008; Ishikawaa et al. 2012; Nakashima \& Nakatsuji 2013; Nakashima et al. 2013), the most accurate full linelist seems to be that from Coppola et al. (2011). With the aim of calculating the radiative cooling function, they used the PEC and the DMC generated in Esry \& Sadeghpour (1999) using an approach that resorts to a particular adiabatic formalism that generates isotopic electronic splitting instead of accounting for the ab initio DBOC.

Here, the most accurate PECs referred to in Section 3 corrected for DBOC and relativistic effects are used, and the results include non-adiabatic corrections obtained with the method described in Section 4. This yields a complete linelist for $\mathrm{HD}^{+}$that is likely the most accurate to date, and a very accurate full linelist for HD.

Table 1 presents pure vibrational transition energies for $\mathrm{HD}^{+}$. The results are compared to quasi-exact results from Balint-Kurti et al. (1990). The improvement in comparison with the linelist presented in Coppola et al. (2011) is remarkable. The deviations between the present linelist and that of Coppola et al. (2011) of dozens of $\mathrm{cm}^{-1}$ can probably be attributed to the inaccuracy of their non-adiabatic corrections, as well as the procedure of using the adiabatic corrections taken from Esry \& Sadeghpour (1999), which are not equivalent to the ab initio DBOC. On the other hand, the larger error that 
Table 2

Computed Transition Frequencies for $\mathrm{HD}^{+}$Compared (Observed-Calculated) with Experimental Data

\begin{tabular}{|c|c|c|c|c|c|c|c|}
\hline \multirow[b]{2}{*}{$v^{\prime}-v^{\prime \prime}$} & \multirow[b]{2}{*}{$J$} & \multicolumn{3}{|c|}{$P(J)$} & \multicolumn{3}{|c|}{$R(J)$} \\
\hline & & Experiment $^{\mathrm{a}}$ & Coppola $^{\mathrm{b}}$ & This Work & Experiment $^{\mathrm{a}}$ & Coppola $^{\mathrm{b}}$ & This Work \\
\hline \multirow[t]{4}{*}{$\overline{1-0}$} & 0 & & & & & & \\
\hline & 1 & 1869.134 & 1.036 & 0.020 & & & \\
\hline & 2 & 1823.533 & 0.978 & 0.021 & & & \\
\hline & 3 & 1776.459 & 0.928 & 0.023 & & & \\
\hline $2-1$ & 0 & & & & 1856.778 & 0.556 & 0.011 \\
\hline \multirow[t]{3}{*}{$3-2$} & 0 & & & & 1761.616 & -0.664 & 0.006 \\
\hline & 1 & & & & 1797.522 & -0.433 & 0.005 \\
\hline & 2 & 1642.108 & -0.955 & 0.011 & 1831.083 & -0.162 & 0.004 \\
\hline \multirow[t]{6}{*}{$17-14$} & 0 & & & & 1813.852 & 23.954 & -0.173 \\
\hline & 1 & 1782.772 & 19.650 & -0.169 & 1820.209 & 25.416 & -0.173 \\
\hline & 2 & & & & 1820.199 & 26.306 & -0.178 \\
\hline & 3 & & & & 1813.644 & 26.581 & -0.182 \\
\hline & 4 & & & & 1800.358 & 26.224 & -0.186 \\
\hline & 5 & & & & 1780.145 & 25.246 & -0.191 \\
\hline \multirow[t]{6}{*}{$17-15$} & 1 & 1092.124 & 16.782 & -0.115 & & & \\
\hline & 2 & 1071.561 & 14.285 & -0.115 & & & \\
\hline & 3 & 1047.239 & 11.692 & -0.114 & & & \\
\hline & 5 & 987.917 & 6.688 & -0.115 & & & \\
\hline & 6 & 953.180 & 4.504 & -0.117 & & & \\
\hline & 7 & & & & 1078.853 & 20.126 & -0.149 \\
\hline \multirow[t]{7}{*}{$18-16$} & 0 & & & & 926.490 & 29.362 & -0.152 \\
\hline & 1 & 901.565 & 25.509 & -0.148 & 932.224 & 30.542 & -0.156 \\
\hline & 2 & 882.731 & 23.026 & -0.150 & 933.213 & 31.124 & -0.161 \\
\hline & 3 & & & & 929.247 & 31.063 & -0.168 \\
\hline & 4 & & & & 920.100 & 30.340 & -0.177 \\
\hline & 5 & & & & 905.519 & 28.963 & -0.191 \\
\hline & 6 & & & & 885.218 & 26.979 & -0.208 \\
\hline \multirow[t]{4}{*}{$20-17$} & 0 & & & & 918.102 & 59.974 & -0.927 \\
\hline & 1 & 900.488 & 56.898 & -0.889 & 915.476 & 60.207 & -1.006 \\
\hline & 2 & 880.668 & 54.212 & -0.920 & 904.833 & 59.533 & -1.145 \\
\hline & 3 & & & & 885.749 & 58.000 & -1.388 \\
\hline \multirow[t]{5}{*}{$21-17$} & 0 & & & & 998.533 & 83.009 & -8.459 \\
\hline & 1 & 984.330 & 80.537 & -7.935 & 988.993 & 81.980 & -9.553 \\
\hline & 2 & & & & 967.811 & 79.404 & -11.235 \\
\hline & 3 & 927.192 & 72.691 & -9.543 & $\ldots$ & $\ldots$ & $\ldots$ \\
\hline & 4 & 882.523 & 67.049 & -11.223 & $\ldots$ & $\ldots$ & $\ldots$ \\
\hline \multirow[t]{5}{*}{$22-17$} & 0 & & & & 1006.965 & 86.886 & -14.720 \\
\hline & 1 & 994.112 & 84.685 & -14.605 & & & \\
\hline & 2 & 969.530 & 81.191 & -14.714 & & & \\
\hline & $\mathrm{rms}$ & & 44.994 & 6.120 & & 44.790 & 4.434 \\
\hline & $\mathrm{rms}^{*}$ & & 10.380 & 0.098 & & 19.885 & 0.141 \\
\hline
\end{tabular}

Notes. All units are $\mathrm{cm}^{-1}$. rms ${ }^{*}$ discards states with $v$ larger or equal to 18 , for which the average $R$ approaches/exceeds the avoided-crossing region.

${ }^{\text {a }}$ Carrington et al. (1989).

${ }^{\mathrm{b}}$ Coppola et al. (2011).

appears for higher states and affects both their and our calculations is most certainly due to an avoided crossing similar to that appearing in $\mathrm{H}_{2}^{+}$at $R \simeq 6$ au (Jiang et al. 2017). The error becomes particularly significant for vibrational levels with a $v$ equal and larger than 18 .

Table 2 presents energies of the rovibrational transitions of the $P$ and $R$ branches of $\mathrm{HD}^{+}$for which experimental values are available. The transitions are compared with the linelist taken from Coppola et al. (2011). The present results are clearly superior, with some low-lying transitions having an accuracy of less than $0.1 \mathrm{~cm}^{-1}$.

Shen et al. (2012) resolved the hyperfine structure of the fundamental rotational transition $(0,0)-(0,1)$, which turns to extend over +0.0100 and $-0.0233 \mathrm{~cm}^{-1}$ around the spinless theoretical value of $43.861201872 \mathrm{~cm}^{-1}$ from Korobov (see reference 23 in
Shen et al. 2012 and Korobov 2006, 2008). Remarkably, the present result for this transition, $43.862873992 \mathrm{~cm}^{-1}$, deviates by only $0.0017 \mathrm{~cm}^{-1}$ from Korobov's result.

Carrington et al. (1992) performed measurements and accurate calculations (within $0.001 \mathrm{~cm}^{-1}$ ) for some transitions between bound and quasibound states. In Table 3 the performances of the two linelists are compared for these transitions, showing again the superiority of the present results. A look to the rms deviations shows that the quality of the present results is kept for the quasibound states.

For applications in astrophysics it is important that the linelist include the quasibound states, which are frequently necessary for understanding many physical and chemical molecular properties. On the other hand, some transitions involving these states have lifetimes that are too small due to 
Table 3

Bound to Quasibound Computed Transition Frequencies for $\mathrm{HD}^{+}$Compared (Observed-Calculated) with Experimental Data

\begin{tabular}{|c|c|c|c|c|c|c|c|}
\hline \multirow[b]{2}{*}{$v^{\prime}-v^{\prime \prime}$} & \multirow[b]{2}{*}{$J$} & \multicolumn{3}{|c|}{$P(J)$} & \multicolumn{3}{|c|}{$R(J)$} \\
\hline & & Experiment $^{\mathrm{a}}$ & Coppola $^{\mathrm{b}}$ & This Work & Experiment $^{\mathrm{a}}$ & Coppola $^{\mathrm{b}}$ & This Work \\
\hline $1-0$ & 44 & & & & 1022.667 & -0.202 & 0.137 \\
\hline $2-1$ & 42 & & & & 1010.735 & -0.161 & 0.109 \\
\hline $3-2$ & 40 & & & & 999.144 & -0.108 & 0.081 \\
\hline $4-3$ & 38 & & & & 986.392 & -0.047 & 0.054 \\
\hline $13-10$ & 24 & 1087.426 & 0.052 & 0.004 & & & \\
\hline $14-11$ & 22 & 1047.787 & 0.145 & -0.050 & & & \\
\hline $14-12$ & 20 & & & & 1034.779 & 0.426 & -0.043 \\
\hline $15-13$ & 18 & & & & 949.049 & 0.660 & -0.118 \\
\hline $15-12$ & 20 & 994.682 & 0.211 & -0.132 & & & \\
\hline \multirow[t]{4}{*}{$17-15$} & 7 & & & & 1078.853 & 20.126 & -0.149 \\
\hline & 8 & & & & 1051.735 & 17.916 & -0.156 \\
\hline & 10 & & & & 978.917 & 12.766 & -0.183 \\
\hline & 11 & & & & 932.229 & 10.139 & -0.211 \\
\hline $16-13$ & 18 & 926.176 & 0.284 & -0.265 & & & \\
\hline \multirow{3}{*}{$19-16$} & 6 & 1036.961 & 27.789 & -0.398 & & & \\
\hline & 7 & 983.693 & 23.9367 & -0.456 & & & \\
\hline & $\mathrm{rms}$ & & 14.974 & 0.276 & & 9.962 & 0.134 \\
\hline
\end{tabular}

Notes. All units are $\mathrm{cm}^{-1}$.

${ }^{\text {a }}$ Carrington et al. (1992).

${ }^{\mathrm{b}}$ Coppola et al. (2011).

Table 4

Computed Transition Frequencies for HD Compared with Experimental Data (Observed-Calculated)

\begin{tabular}{|c|c|c|c|c|c|c|c|}
\hline \multirow[b]{2}{*}{$v^{\prime}-v^{\prime \prime}$} & \multirow[b]{2}{*}{$J$} & \multicolumn{3}{|c|}{$P(J)$} & \multicolumn{3}{|c|}{$R(J)$} \\
\hline & & Experiment $^{\mathrm{a}}$ & Pachucki $^{\text {b }}$ & This Work & Experiment $^{\mathrm{a}}$ & Pachucki $^{\text {b }}$ & This Work \\
\hline \multirow[t]{5}{*}{$\overline{1-0}$} & 0 & & & & 3717.532 & -0.0003 & 0.048 \\
\hline & 1 & 3542.932 & -0.0005 & 0.055 & 3798.455 & 0.0035 & 0.050 \\
\hline & 2 & 3450.463 & -0.001 & 0.060 & 3874.357 & 0.0038 & 0.049 \\
\hline & 3 & 3355.361 & -0.009 & 0.058 & 3944.720 & 0.0011 & 0.047 \\
\hline & 4 & & & & 4009.088 & 0.0012 & 0.049 \\
\hline \multirow[t]{4}{*}{$4-0$} & 0 & & & & 13551.065 & -0.0117 & 0.003 \\
\hline & 1 & 13387.646 & -0.0149 & 0.000 & 13609.664 & -0.0121 & 0.002 \\
\hline & 2 & 13283.993 & -0.0145 & 0.007 & 13652.215 & -0.0032 & 0.012 \\
\hline & 3 & & & & 13678.322 & -0.0039 & 0.014 \\
\hline \multirow[t]{5}{*}{$5-0$} & 0 & & & & 16486.537 & -0.0115 & -0.090 \\
\hline & 1 & 16326.791 & -0.0138 & -0.093 & 16537.816 & -0.0017 & -0.087 \\
\hline & 2 & 16219.473 & -0.0063 & -0.082 & 16569.404 & 0.0044 & -0.088 \\
\hline & 3 & & & & 16581.008 & 0.0501 & -0.050 \\
\hline & $\mathrm{rms}$ & & 0.0105 & 0.060 & & 0.0152 & 0.054 \\
\hline
\end{tabular}

Notes. All units are $\mathrm{cm}^{-1}$.

${ }^{a}$ Chuang \& Zare (1987).

b Pachucki \& Komasa (2010).

predissociation Carrington et al. (1992, 1993). For the convenience of the reader, in the tar.gz package these states are separated and located at the bottom of the tables.

Moving to HD, the reference work from Wolniewicz (1995) displays very accurate energy values and transitions, though no attempt to generate the full linelist or complete set of Einstein A-coefficients has been made. An old linelist from Abgrall et al. shows an attempt to include electric quadrupole transitions but presents large deviations of about $1 \mathrm{~cm}^{-1}$ in the transitions. The more recent linelist for HD is from Pachucki \& Komasa (2010), with the full data set displayed in the HITRAN Database (Rothman et al. 2017). It includes nonadiabatic, relativistic, QED, and smaller effects, thus presenting extremely accurate transition values within $0.01 \mathrm{~cm}^{-1}$, but no transitions involving quasibond states.

Table 4 shows energies of some rovibrational transitions obtained here compared with experimental values, resulting in a very good rms deviation of $0.05 \mathrm{~cm}^{-1}$, which is consistent with the other applications despite being worse than that in Pachucki \& Komasa (2010). It is not easy to explain this difference at this level of accuracy. Apparently, their quasi-exact approach for $\mathrm{H}_{2}$ bound states in Pachucki \& Komasa (2009) is easier to be extend 


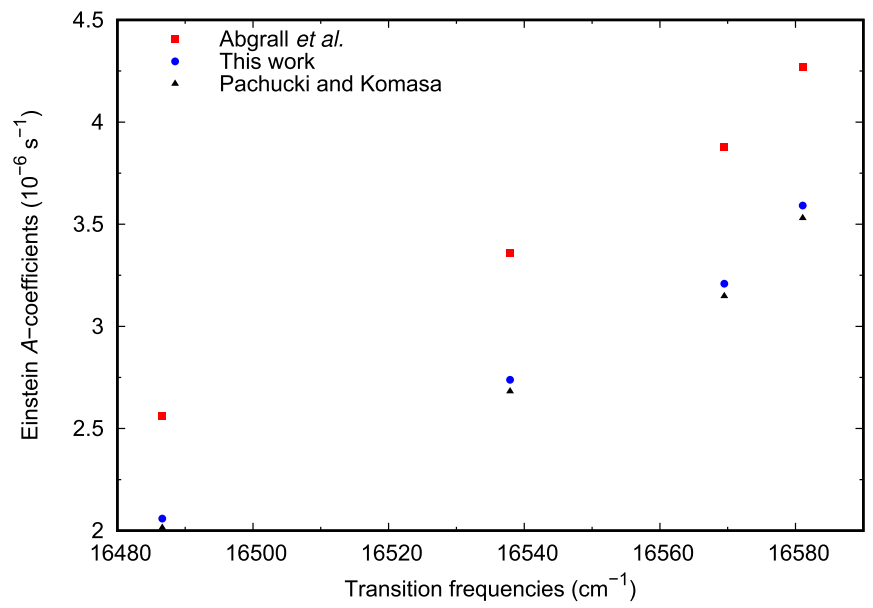

Figure 2. Comparison of the Einstein A-coefficients for the (5-0) band of the $R$-branch of HD.

to HD (Pachucki \& Komasa 2010) with a perturbative approach. Furthermore, some other factors influence the observed differences besides the inclusion of QED corrections. While the present non-adiabatic corrections are fully based on effective nuclear masses taken from the electronic density, part of the nonadiabatic corrections in Pachucki \& Komasa (2010) is in the potential, while their vibrational effective mass assumes "nonphysical" values larger than the atomic mass, namely the sum of the masses of the atoms, for some values of $R$. On the other hand, the present work includes quasibound states in the linelist as well as A-coefficients, absent in Pachucki \& Komasa (2010).

The full lists of Einstein A-coefficients for $\mathrm{HD}^{+}$and $\mathrm{HD}$ can be found in the tar.gz package. Figures 1 and 2 display selected examples concerning comparison with previous calculations. It has been shown that the A-coefficients are affected little by the nonadiabatic effects (Diniz et al. 2016), but they are sensitive to the quality of the wavefunctions and energies, so that they are expected to differ from the previously reported values. Figure 1 compares the present values of A-coefficients for the (18-16) band of the R-branch of $\mathrm{HD}^{+}$with those from Coppola et al. (2011). Small but significant differences can be seen. For the (5-0) band of the R-branch of HD (Figure 2), the present values are compared with the data from Abgrall et al. (1982) and Pachucki \& Komasa (2010). As expected, the present transition probabilities are very close but not exactly equal to those from Pachucki \& Komasa (2010). The calculation of the high-resolution spectroscopic parameters of the studied molecules should profit from the accuracy improvements implemented in the present work.

\section{2. $\mathrm{HeH}^{+}$and Isotopologues}

$\mathrm{HeH}^{+}$is the smallest heteronuclear molecule and is supposed to be the first molecule formed in the primordial universe. Its recent discovery in space represents a spectacular progress for the field of molecular astrophysics (Güsten et al. 2019). In Roberge \& Dalgarno (1982) one can find a study of the formation and destruction of $\mathrm{HeH}^{+}$in astrophysical plasmas. There are six stable isotopologues of the system, ${ }^{4} \mathrm{HeH}^{+},{ }^{4} \mathrm{HeD}^{+},{ }^{4} \mathrm{HeT}^{+},{ }^{3} \mathrm{HeH}^{+}$, ${ }^{3} \mathrm{HeD}^{+}$, and ${ }^{3} \mathrm{HeT}^{+}$with the first being the most prevalent. Some sets of rovibrational and pure rotational transitions obtained using different experimental techniques were collected (Coxon \& Hajigeorgiou 1999) and have been used for comparison by theoreticians. In this work they are used too.
Table 5

Rotation-vibration Frequencies for ${ }^{4} \mathrm{HeH}^{+}$(Observed-Calculated), Pachucki \& Komasa (2012), and Present Work without and with QED, Compared with Experimental Data

\begin{tabular}{lccccc}
\hline \hline$\nu$ & $J$ & Experiment $^{\mathrm{a}}$ & Pachucki & This Work & This Work QED \\
\hline 1 & 1 & 67.053 & 0.004 & -0.002 & -0.001 \\
& 2 & 200.769 & 0.010 & -0.007 & -0.005 \\
& 3 & 400.379 & 0.020 & -0.013 & -0.008 \\
& 4 & 664.732 & 0.032 & -0.025 & -0.016 \\
& 5 & 992.324 & 0.051 & -0.034 & -0.021 \\
& 6 & 1381.287 & 0.067 & -0.051 & -0.033 \\
& 7 & 1829.447 & 0.093 & -0.064 & -0.040 \\
& 0 & 2910.958 & 0.006 & -0.010 & 0.007 \\
& 1 & 2972.574 & 0.009 & -0.013 & 0.005 \\
& 2 & 3095.427 & 0.014 & -0.017 & 0.003 \\
& 3 & 3278.761 & 0.023 & -0.024 & -0.002 \\
& 4 & 3521.456 & 0.036 & -0.032 & -0.007 \\
& 5 & 3822.029 & 0.050 & -0.044 & -0.015 \\
& 6 & 4178.661 & 0.067 & -0.057 & -0.024 \\
& 7 & 4589.196 & 0.079 & -0.079 & -0.041 \\
& 8 & 5051.199 & 0.107 & -0.089 & -0.046 \\
\hline
\end{tabular}

Note. All units are $\mathrm{cm}^{-1}$.

${ }^{\text {a }}$ Perry et al. (2014).

Comparison of the present results is made in Table 5 with some recent experimental data from Perry et al. (2014), obtained with high precision sub-Doppler infrared spectroscopy, as well as to the ${ }^{4} \mathrm{HeH}^{+}$calculations in Pachucki \& Komasa (2012). It is notable that the present results are systematically larger while, in contrast, Pachucki \& Komasa (2012) values are systematically lower than the experimental results. Since Pachucki \& Komasa (2012) use the same approach of their application to $\mathrm{HD}$, this becomes an opportunity to check our supposition in the previous section that the differences of the two approaches lye mainly in the different non-adiabatic corrections. With this goal, the QED corrections from Pachucki \& Komasa (2012) are added to the present PEC and a corresponding set of transition energies is produced, shown in the last column of Table 5. The improvement in the accuracy of the present results in the second decimal place in $\mathrm{cm}^{-1}$ is notable, but only a few values become lower than the experimental ones. The differences from Pachucki \& Komasa (2012) diminish but still keep in the second decimal place thus reinforcing our supposition that their main source is the different non-adiabatic approaches and that the superior performance of Pachucky and Komasa's method for HD is probably due to its fitness to this particular system. Note also that, different from the HD case, even the present results without QED contributions are more accurate than those in Pachucki \& Komasa (2012). Thus, for further comparison, only the works displaying large number of transitions and different isotopologues are kept.

Contrary to the previously described systems, very accurate linelists exist for $\mathrm{HeH}^{+}$and its isotopologues such that the present results obtained with the new PECs and the $R$-dependent vibrational masses can be gauged. The present results are compared with two previous calculations. The PEC from Kolos \& Peek (1976) corrected for DBOC in Bishop \& Cheung (1979), but without the relativistic corrections, was used in the rovibrational calculations performed in Engel et al. (2005). To simulate non-adiabatic effects they tried various effective 
reduced nuclear masses, but in the end chose the dissociation reduced mass, as it produced results that agreed best with the experimental results. Here, only their results obtained with the latter choice of reduced mass are used for comparison. While their results for ${ }^{4} \mathrm{HeH}^{+}$are explicitly presented in Engel et al. (2005), those for the other isotopologues are found in the ExoMol compilation (Tennyson et al. 2016). In Tung et al. (2012) a very accurate $\mathrm{HeH}^{+}$PEC from Pachucki (2012) augmented with DBOCs, but without the relativistic corrections, was used in the calculations. To account for non-adiabatic effects, they also used a constant vibrational reduced mass obtained by the minimization of the difference between their transition energy of the lowest pure vibrational transition of ${ }^{4} \mathrm{HeH}^{+}$and that of their previous non-BO calculation. The difference of their lowest calculated transition and the experimental transition then becomes zero. This optimized reduced mass was also used for the other states without reoptimization.

The procedure of Tung et al. (2012) improved upon the results of Engel et al. (2005) by about one order of magnitude. An analysis of Table 1 or 2 in Engel et al. (2005) shows clearly that the significant differences between the two sets of results cannot be attributed to only the non-adiabatic corrections, but are result of the improvements of both the BO PEC and the DBOC by Tung et al. The present PECs are further improved relative to the PECs of Tung et al. through the inclusion of relativistic corrections.

The comparison presented in Table 6 for purely rotational transitions of ${ }^{4} \mathrm{HeH}^{+}$provides some interesting information. One can see that the present results and those of Engel et al. and Tung et al. have approximately the same quality, as determined by comparing the rms deviations. In fact, it becomes clear that any reasonable rotational mass works well and the inclusion of the relativistic corrections (this work) changes the results expressed in $\mathrm{cm}^{-1}$ only at the third figure after the decimal point.

The most significant improvement of the results is achieved in the present approach due to the use of the improved vibrational masses, as shown in Tables 7-10. For ${ }^{4} \mathrm{HeH}^{+}$(Table 7) and for ${ }^{4} \mathrm{HeD}^{+}$(Table 8), for which the most comprehensive comparison can be performed due to a large number of experimental transitions, the present rms deviations are one order of magnitude smaller, on average, than those of Engel et al., and about half of those of Tung et al. For ${ }^{3} \mathrm{HeH}^{+}$(Table 9) and ${ }^{3} \mathrm{HeD}^{+}$(Table 10) the improvement is not as impressive, though still noticeable. This mostly results from the small number of available experimental transition energies that can be used in the comparison.

\section{Discussion}

Present-day experimental spectroscopy of primordial molecules has accuracy better than $10^{-2} \mathrm{~cm}^{-1}$. Experimental data, however, are limited to few transitions and need to be supplemented with theoretical computations of high accuracy, which are fundamental for those working with molecular astrophysics and cosmology.

Once very accurate BO PECs (PESs) are calculated, they can be augmented by a hierarchy of the corrections that make the calculation of the rovibrational levels more accurate. These corrections are: (i) adiabatic (DBOC; changing the transition energies by a few $\mathrm{cm}^{-1}$ ), (ii) non-adiabatic (changing the results of tenths to few units of $\mathrm{cm}^{-1}$ ), and (iii) relativistic plus QED (affecting the results by fractions of $\mathrm{cm}^{-1}$ ). While DBOC, relativistic, and QED corrections are directly included in the PECs, the non-adiabatic corrections are usually included at the level of the nuclear equation that is solved for the rovibrational
Table 6

Computed Pure Rotational Transition Frequencies for ${ }^{4} \mathrm{HeH}^{+}$(ObservedCalculated), Engel et al. (2005), Tung et al. (2012), and the Present Work, Compared with Experimental Data

\begin{tabular}{|c|c|c|c|c|c|c|c|}
\hline$v$ & $J^{\prime \prime}$ & $J^{\prime}$ & Experiment & Engel & Tung & This work & \\
\hline \multirow[t]{17}{*}{0} & 0 & 1 & $67.053^{\mathrm{a}}$ & 0.002 & -0.001 & -0.002 & \\
\hline & 1 & 2 & $133.717^{\mathrm{a}}$ & 0.003 & -0.002 & -0.004 & \\
\hline & 6 & 7 & $448.160^{\mathrm{b}}$ & 0.016 & -0.006 & -0.012 & \\
\hline & 10 & 11 & $657.221^{\mathrm{c}}$ & 0.005 & -0.016 & -0.022 & \\
\hline & 11 & 12 & $701.317^{\mathrm{c}}$ & 0.013 & -0.009 & -0.014 & \\
\hline & 12 & 13 & $741.706^{\mathrm{c}}$ & 0.007 & -0.014 & -0.018 & \\
\hline & 13 & 14 & $778.224^{\mathrm{c}}$ & 0.002 & -0.018 & -0.022 & \\
\hline & 14 & 15 & $810.708^{c}$ & -0.005 & -0.023 & -0.026 & \\
\hline & 15 & 16 & $839.010^{\mathrm{c}}$ & -0.008 & -0.027 & -0.027 & \\
\hline & 16 & 17 & $862.984^{c}$ & -0.011 & -0.028 & -0.027 & \\
\hline & 17 & 18 & $882.475^{\mathrm{c}}$ & -0.015 & -0.032 & -0.028 & \\
\hline & 18 & 19 & $897.334^{\mathrm{c}}$ & -0.005 & -0.022 & -0.015 & \\
\hline & 20 & 21 & $912.242^{\mathrm{c}}$ & -0.012 & -0.028 & -0.016 & \\
\hline & 21 & 22 & $911.704^{\mathrm{c}}$ & -0.011 & -0.028 & -0.012 & \\
\hline & 23 & 24 & $891.888^{d}$ & -0.024 & -0.035 & -0.002 & QB \\
\hline & 24 & 25 & $870.298^{\mathrm{d}}$ & -0.025 & -0.038 & -0.005 & QQ \\
\hline & 25 & 26 & $837.180^{\mathrm{d}}$ & -0.042 & -0.038 & 0.007 & QQ \\
\hline \multirow[t]{14}{*}{1} & 10 & 11 & $598.829^{c}$ & 0.009 & -0.016 & -0.025 & \\
\hline & 11 & 12 & $637.767^{\mathrm{c}}$ & 0.014 & -0.014 & -0.023 & \\
\hline & 12 & 13 & $672.989^{c}$ & 0.018 & -0.103 & -0.019 & \\
\hline & 13 & 14 & $704.270^{c}$ & 0.009 & -0.022 & -0.029 & \\
\hline & 14 & 15 & $731.430^{c}$ & 0.012 & -0.019 & -0.024 & \\
\hline & 15 & 16 & $754.235^{\mathrm{c}}$ & 0.007 & -0.022 & -0.026 & \\
\hline & 16 & 17 & $772.464^{\mathrm{c}}$ & 0.007 & -0.018 & -0.020 & \\
\hline & 17 & 18 & $785.837^{\mathrm{c}}$ & 0.009 & -0.013 & -0.012 & \\
\hline & 18 & 19 & $793.997^{\mathrm{c}}$ & -0.003 & -0.019 & -0.015 & \\
\hline & 19 & 20 & $796.490^{c}$ & -0.010 & -0.019 & -0.012 & \\
\hline & 20 & 21 & $792.616^{\mathrm{d}}$ & -0.020 & -0.021 & -0.010 & QB \\
\hline & 21 & 22 & $781.245^{\mathrm{e}}$ & -0.042 & -0.033 & -0.008 & QQ \\
\hline & 22 & 23 & $760.340^{\mathrm{d}}$ & -0.033 & -0.022 & 0.003 & QQ \\
\hline & 23 & 24 & $724.933^{c}$ & -0.063 & -0.016 & 0.015 & \\
\hline \multirow[t]{10}{*}{2} & 13 & 14 & $627.320^{c}$ & 0.002 & -0.014 & -0.022 & \\
\hline & 14 & 15 & $648.324^{\mathrm{c}}$ & 0.005 & -0.011 & -0.018 & \\
\hline & 15 & 16 & $664.559^{c}$ & 0.003 & -0.014 & -0.020 & \\
\hline & 16 & 17 & $675.609^{c}$ & 0.003 & -0.013 & -0.016 & \\
\hline & 17 & 18 & $680.895^{\mathrm{c}}$ & -0.001 & -0.014 & -0.015 & \\
\hline & 18 & 19 & $679.586^{\mathrm{c}}$ & -0.011 & -0.016 & -0.014 & \\
\hline & 19 & 20 & $670.340^{c}$ & -0.023 & -0.012 & -0.005 & \\
\hline & 20 & 21 & $650.613^{\mathrm{d}}$ & -0.034 & 0.007 & 0.008 & QB \\
\hline & $\mathrm{rms}^{\mathrm{f}}$ & & & 0.015 & 0.026 & 0.019 & \\
\hline & $\mathrm{rms}^{\mathrm{g}}$ & & & 0.019 & 0.026 & 0.018 & \\
\hline
\end{tabular}

Notes. QB denotes transitions between a quasibound and a bound state and QQ denotes transitions between two quasibound states. All units are $\mathrm{cm}^{-1}$.

a Matsushima et al. (1997).

${ }^{\mathrm{b}}$ Liu et al. (1987).

${ }^{\text {c }}$ Liu \& Davies (1997a).

${ }^{\mathrm{d}}$ Liu \& Davies (1997b).

e Hoyland (1967).

${ }^{\mathrm{f}}$ rms calculated neglecting $\mathrm{QB}$ and QQ transitions.

${ }^{\mathrm{g}} \mathrm{rms}$ calculated including QB and QQ transitions.

energies. Furthermore, the relativistic and QED corrections can be positive or negative, depending on the rovibrational quantum numbers. It is possible that some results appearing in the linelists may be very close to the experiment, but others may be quite inaccurate. This problem can only be overcome using truly accurate non-adiabatic corrections. Corrections due to rotational masses also amount for about $10^{-1} \mathrm{~cm}^{-1}$ and can be reliably evaluated with an empirical procedure. The major effect that needs to be accounted for in order to reach the point 
Table 7

Computed Rovibrational Transition Frequencies for ${ }^{4} \mathrm{HeH}^{+}$(Observed-Calculated), Engel et al. (2005), Tung et al. (2012), and the Present Work, Compared with Experimental Data

\begin{tabular}{|c|c|c|c|c|c|c|c|c|c|c|c|}
\hline \multirow[b]{2}{*}{$v^{\prime}-v^{\prime \prime}$} & \multirow[b]{2}{*}{$J$} & \multicolumn{4}{|c|}{$P(J)$} & \multicolumn{4}{|c|}{$R(J)$} & \multirow[b]{2}{*}{ This Work } & \\
\hline & & Experiment & Engel & Tung & This Work & & Experiment & Engel & Tung & & \\
\hline \multirow[t]{13}{*}{$1-0$} & 0 & & & & & & $2972.573^{\mathrm{a}}$ & -0.213 & -0.061 & -0.014 & \\
\hline & 1 & $2843.903^{\mathrm{a}}$ & -0.216 & -0.060 & -0.010 & & $3028.375^{\mathrm{a}}$ & -0.212 & -0.060 & -0.014 & \\
\hline & 2 & $2771.806^{\mathrm{a}}$ & -0.215 & -0.056 & -0.005 & & $3077.992^{\mathrm{a}}$ & -0.216 & -0.061 & -0.017 & \\
\hline & 3 & $2695.050^{\mathrm{a}}$ & -0.218 & -0.053 & -0.001 & & $3121.077^{\mathrm{a}}$ & -0.220 & -0.061 & -0.019 & \\
\hline & 4 & $2614.030^{\mathrm{a}}$ & -0.223 & -0.051 & 0.001 & & $3157.297^{\mathrm{a}}$ & -0.223 & -0.060 & -0.020 & \\
\hline & 5 & $2529.134^{\mathrm{b}}$ & -0.228 & -0.048 & 0.004 & & $3186.337^{\mathrm{b}}$ & -0.230 & -0.062 & -0.023 & \\
\hline & 6 & $2440.742^{\mathrm{b}}$ & -0.232 & -0.044 & 0.007 & & $3207.909^{\mathrm{b}}$ & -0.226 & -0.064 & -0.028 & \\
\hline & 7 & & & & & & $3221.752^{b}$ & -0.228 & -0.060 & -0.025 & \\
\hline & 9 & $2158.140^{\mathrm{c}}$ & -0.240 & -0.032 & 0.015 & & & & & & \\
\hline & 10 & $2059.210^{c}$ & -0.238 & -0.029 & 0.016 & & & & & & \\
\hline & 11 & $1958.388^{\mathrm{c}}$ & -0.234 & -0.027 & 0.016 & & & & & & \\
\hline & 12 & $1855.905^{\mathrm{d}}$ & -0.233 & -0.029 & 0.011 & & & & & & \\
\hline & 13 & $1751.971^{\mathrm{d}}$ & -0.221 & -0.025 & 0.011 & & & & & & \\
\hline \multirow[t]{14}{*}{$2-1$} & 0 & & & & & & $2660.284^{\mathrm{e}}$ & -0.017 & -0.063 & -0.024 & \\
\hline & 1 & $2542.531^{\mathrm{e}}$ & -0.017 & -0.063 & -0.021 & & $2710.566^{\mathrm{e}}$ & -0.021 & -0.063 & -0.026 & \\
\hline & 2 & $2475.814^{\mathrm{e}}$ & -0.019 & -0.062 & -0.019 & & $2754.624^{\mathrm{b}}$ & -0.031 & -0.067 & -0.031 & \\
\hline & 3 & & & & & & $2792.110^{\mathrm{b}}$ & -0.039 & -0.068 & -0.034 & \\
\hline & 4 & & & & & & $2822.683^{\mathrm{b}}$ & -0.047 & -0.066 & -0.034 & \\
\hline & 5 & $2248.854^{\mathrm{c}}$ & -0.028 & -0.047 & -0.002 & & $2846.009^{b}$ & -0.060 & -0.067 & -0.037 & \\
\hline & 6 & $2165.485^{\mathrm{c}}$ & -0.040 & -0.046 & -0.002 & & $2861.786^{\mathrm{b}}$ & -0.053 & -0.061 & -0.033 & \\
\hline & 7 & $2078.841^{\mathrm{c}}$ & -0.067 & -0.048 & -0.003 & & $2869.690^{b}$ & -0.079 & -0.069 & -0.043 & \\
\hline & 8 & $1989.251^{\mathrm{c}}$ & -0.069 & -0.042 & 0.002 & & $2869.478^{\mathrm{b}}$ & -0.062 & -0.041 & -0.017 & \\
\hline & 9 & $1896.992^{d}$ & -0.092 & -0.047 & -0.004 & & & & & & \\
\hline & 10 & $1802.349^{\mathrm{d}}$ & -0.105 & -0.043 & -0.001 & & & & & & \\
\hline & 11 & $1705.543^{d}$ & -0.117 & -0.036 & 0.003 & & & & & & \\
\hline & 19 & $862.529^{f}$ & -0.156 & 0.026 & 0.035 & & & & & & \\
\hline & 20 & $745.624^{f}$ & -0.158 & 0.028 & 0.032 & & & & & & \\
\hline \multirow[t]{7}{*}{$3-2$} & 4 & & & & & & $2484.912^{\mathrm{c}}$ & -0.039 & -0.053 & -0.028 & \\
\hline & 5 & $1966.356^{\mathrm{c}}$ & -0.023 & -0.062 & -0.022 & & $2501.941^{\mathrm{c}}$ & -0.045 & -0.053 & -0.030 & \\
\hline & 6 & & & & & & $2511.188^{\mathrm{c}}$ & -0.055 & -0.054 & -0.033 & \\
\hline & 8 & & & & & & $2504.914^{\mathrm{c}}$ & -0.076 & -0.046 & -0.028 & \\
\hline & 9 & & & & & & $2488.632^{\mathrm{c}}$ & -0.083 & -0.043 & -0.027 & \\
\hline & 17 & $833.640^{f}$ & -0.147 & 0.001 & 0.020 & & & & & & \\
\hline & 18 & $719.769^{f}$ & -0.158 & 0.019 & 0.034 & & & & & & \\
\hline \multirow[t]{2}{*}{$5-4$} & 11 & $901.963^{\mathrm{f}}$ & -0.102 & -0.029 & 0.012 & & & & & & \\
\hline & 12 & $807.806^{\mathrm{f}}$ & -0.127 & -0.035 & 0.005 & & & & & & \\
\hline \multirow[t]{3}{*}{$6-5$} & 8 & $863.378^{f}$ & -0.091 & -0.050 & 0.001 & & & & & & \\
\hline & 9 & $782.925^{f}$ & -0.093 & -0.038 & 0.013 & & & & & & \\
\hline & 12 & & & & & & $979.904^{\mathrm{g}}$ & -0.248 & 0.065 & 0.050 & QB \\
\hline \multirow[t]{2}{*}{$7-6$} & 4 & $817.337^{f}$ & -0.172 & -0.020 & 0.040 & & & & & & \\
\hline & 5 & $760.367^{\mathrm{h}}$ & -0.200 & -0.039 & 0.022 & & & & & & \\
\hline \multirow[t]{3}{*}{$7-5$} & 12 & $938.200^{\mathrm{i}}$ & 0.136 & 0.095 & 0.161 & QB & & & & & \\
\hline & \multirow{2}{*}{\multicolumn{2}{|c|}{$\begin{array}{l}\mathrm{rms}^{\mathrm{j}} \\
\mathrm{rms}^{\mathrm{k}}\end{array}$}} & 0.163 & 0.042 & 0.017 & & & 0.140 & 0.060 & 0.028 & \\
\hline & & & 0.162 & 0.045 & 0.033 & & & 0.146 & 0.060 & 0.029 & \\
\hline
\end{tabular}

Notes. QB denotes transitions between a quasibound and a bound state. All units are $\mathrm{cm}^{-1}$.

a Bernath \& Amano (1982).

${ }^{\text {b }}$ Crofton et al. (1989).

${ }^{\mathrm{c}}$ Purder et al. (1992).

d Tolliver et al. (1979).

e Blom et al. (1987).

${ }^{\mathrm{f}}$ Liu \& Davies (1997a).

g Carrington et al. (1983).

h Hoyland (1967).

${ }^{\mathrm{i}}$ Carrington et al. (1981).

$\mathrm{j}$ rms calculated neglecting QB transitions.

${ }^{\mathrm{k}}$ rms calculated including QB transitions.

at which relativistic, QED, and rotational-mass corrections are relevant is the proper accounting for corrections to the vibrational masses.
In the present work, an improved procedure for determining vibrational masses is introduced. The procedure uses ab initio electronic densities and overcomes the problem of heuristically 
Table 8

Computed Rovibrational Transition Frequencies for ${ }^{4} \mathrm{HeD}^{+}$(Observed-Calculated), Engel et al. (2005), Tung et al. (2012), and the Present Work, Compared with Experimental Data

\begin{tabular}{|c|c|c|c|c|c|c|c|c|c|c|c|}
\hline \multirow[b]{2}{*}{$v^{\prime}-v^{\prime \prime}$} & \multirow[b]{2}{*}{$J$} & \multicolumn{4}{|c|}{$P(J)$} & \multicolumn{4}{|c|}{$R(J)$} & \multirow[b]{2}{*}{ This work } & \\
\hline & & Experiment & Engel & Tung & This work & & Experiment & Engel & Tung & & \\
\hline \multirow[t]{11}{*}{$1-0$} & 0 & & & & & & $2348.628^{\mathrm{a}}$ & -0.184 & -0.069 & -0.039 & \\
\hline & 1 & $2269.812^{\mathrm{a}}$ & -0.183 & -0.062 & -0.031 & & $2384.108^{a}$ & -0.180 & -0.066 & -0.037 & \\
\hline & 2 & & & & & & $2416.780^{\mathrm{a}}$ & -0.181 & -0.069 & -0.040 & \\
\hline & 3 & $2181.432^{\mathrm{a}}$ & -0.191 & -0.061 & -0.029 & & $2446.518^{\mathrm{a}}$ & -0.185 & -0.072 & -0.044 & \\
\hline & 4 & $2134.011^{\mathrm{b}}$ & -0.198 & -0.063 & -0.031 & & $2473.202^{\mathrm{a}}$ & -0.184 & -0.071 & -0.044 & \\
\hline & 5 & $2084.633^{\mathrm{b}}$ & -0.202 & -0.060 & -0.029 & & $2496.703^{\mathrm{a}}$ & -0.191 & -0.075 & -0.049 & \\
\hline & 6 & & & & & & $2516.917^{\mathrm{a}}$ & -0.191 & -0.075 & -0.049 & \\
\hline & 7 & & & & & & $2533.732^{\mathrm{a}}$ & -0.193 & -0.075 & -0.050 & \\
\hline & 8 & $1926.132^{\mathrm{b}}$ & -0.212 & -0.052 & -0.021 & & $2547.048^{\mathrm{a}}$ & -0.196 & -0.075 & -0.051 & \\
\hline & 9 & & & & & & $2556.772^{\mathrm{a}}$ & -0.188 & -0.074 & -0.051 & \\
\hline & 10 & & & & & & $2562.812^{\mathrm{a}}$ & -0.186 & -0.075 & -0.053 & \\
\hline \multirow[t]{4}{*}{$2-1$} & 1 & $2088.030^{\mathrm{b}}$ & -0.054 & -0.069 & -0.040 & & & & & & \\
\hline & 3 & & & & & & $2252.028^{b}$ & -0.050 & -0.069 & -0.043 & \\
\hline & 4 & $1959.756^{\mathrm{b}}$ & -0.055 & -0.056 & -0.026 & & & & & & \\
\hline & 5 & & & & & & $2297.086^{\mathrm{b}}$ & -0.057 & -0.072 & -0.048 & \\
\hline \multirow[t]{3}{*}{$3-2$} & 4 & & & & & & $2078.586^{\mathrm{b}}$ & -0.004 & -0.061 & -0.037 & \\
\hline & 5 & & & & & & $2096.816^{\mathrm{b}}$ & -0.021 & -0.070 & -0.046 & \\
\hline & 8 & & & & & & $2131.027^{\mathrm{b}}$ & -0.056 & -0.072 & -0.050 & \\
\hline \multirow[t]{2}{*}{$4-3$} & 9 & & & & & & $1920.660^{\mathrm{b}}$ & -0.029 & -0.061 & -0.037 & \\
\hline & 12 & & & & & & $1899.333^{b}$ & -0.060 & -0.055 & -0.033 & \\
\hline $6-4$ & 22 & $1088.373^{\mathrm{c}}$ & -0.405 & 0.091 & 0.085 & QB & & & & & \\
\hline $6-5$ & 20 & & & & & & $1003.329^{c}$ & -0.263 & 0.066 & 0.041 & QB \\
\hline $7-5$ & 20 & $944.720^{c}$ & -0.429 & 0.100 & 0.103 & QB & & & & & \\
\hline \multirow[t]{4}{*}{ 13-9 } & 4 & & & & & & $1073.475^{\mathrm{c}}$ & 0.298 & -0.013 & 0.158 & QB \\
\hline & 6 & $911.705^{\mathrm{c}}$ & 0.381 & -0.023 & 0.159 & QB & & & & & \\
\hline & & & 0.169 & 0.061 & 0.030 & & & 0.149 & 0.070 & 0.045 & \\
\hline & & & 0.263 & 0.067 & 0.070 & & & 0.167 & 0.068 & 0.056 & \\
\hline
\end{tabular}

Notes. QB denotes transitions between a quasibound and a bound state. All units are $\mathrm{cm}^{-1}$.

${ }^{\mathrm{a}}$ Crofton et al. (1989).

${ }^{b}$ Purder et al. (1992).

${ }^{c}$ Carrington et al. (1983).

${ }^{\mathrm{d}}$ rms calculated neglecting QB transitions.

e rms calculated including QB transitions.

Table 9

Computed Rovibrational Transition Frequencies for ${ }^{3} \mathrm{HeH}^{+}$(Observed-Calculated), Engel et al. (2005), Tung et al. (2012), and the Present Work, Compared with Experimental Data

\begin{tabular}{|c|c|c|c|c|c|c|c|c|c|c|}
\hline \multirow[b]{2}{*}{$v^{\prime}-v^{\prime \prime}$} & \multirow[b]{2}{*}{$J$} & \multicolumn{4}{|c|}{$P(J)$} & \multicolumn{4}{|c|}{$R(J)$} & \\
\hline & & Experiment & Engel & Tung & This work & Experiment & Engel & Tung & This work & \\
\hline \multirow[t]{8}{*}{$1-0$} & 0 & & & & & $3060.433^{\mathrm{a}}$ & -0.271 & -0.080 & -0.037 & \\
\hline & 1 & 2923.680 & -0.279 & -0.078 & -0.033 & $3119.405^{\mathrm{a}}$ & -0.268 & -0.082 & -0.040 & \\
\hline & 2 & 2846.775 & -0.281 & -0.072 & -0.026 & $3171.549^{\mathrm{a}}$ & -0.266 & -0.083 & -0.043 & \\
\hline & 3 & 2764.768 & -0.287 & -0.073 & -0.026 & $3216.468^{\mathrm{a}}$ & -0.265 & -0.084 & -0.047 & \\
\hline & 4 & 2678.113 & -0.290 & -0.069 & -0.023 & $3253.785^{\mathrm{a}}$ & -0.267 & -0.088 & -0.052 & \\
\hline & 5 & 2587.243 & -0.296 & -0.068 & -0.023 & $3283.156^{\mathrm{a}}$ & -0.258 & -0.086 & -0.053 & \\
\hline & 6 & 2492.591 & -0.298 & -0.064 & -0.020 & $3304.247^{\mathrm{a}}$ & -0.254 & -0.088 & -0.057 & \\
\hline & 7 & & & & & $3316.761^{\mathrm{a}}$ & -0.257 & -0.088 & -0.059 & \\
\hline \multirow[t]{3}{*}{$6-5$} & 11 & & & & & $981.322^{\mathrm{b}}$ & -0.208 & 0.123 & 0.096 & QB \\
\hline & $\mathrm{rms}^{\mathrm{c}}$ & & 0.289 & 0.071 & 0.026 & & 0.263 & 0.085 & 0.049 & \\
\hline & $\mathrm{rms}^{\mathrm{d}}$ & & & & & & 0.258 & 0.090 & 0.056 & \\
\hline
\end{tabular}

Notes. QB denotes transitions between a quasibound and a bound state. All units are $\mathrm{cm}^{-1}$

${ }^{a}$ Crofton et al. (1989).

${ }^{b}$ Carrington et al. (1983).

${ }^{\mathrm{c}}$ rms calculated neglecting QB transitions.

${ }^{\mathrm{d}}$ rms calculated including QB transitions. 
Table 10

Computed Rovibrational Transition Frequencies for ${ }^{3} \mathrm{HeD}^{+}$(Observed-Calculated), Engel et al. (2005), Tung et al. (2012), and the Present Work, Compared with Experimental Data

\begin{tabular}{|c|c|c|c|c|c|c|c|c|c|c|c|}
\hline \multirow[b]{2}{*}{$v^{\prime}-v^{\prime \prime}$} & \multirow[b]{2}{*}{$J$} & \multicolumn{4}{|c|}{$P(J)$} & \multicolumn{4}{|c|}{$R(J)$} & \multirow[b]{2}{*}{ This Work } & \\
\hline & & Experiment & Engel & Tung & This Work & & Experiment & Engel & Tung & & \\
\hline \multirow[t]{4}{*}{$1-0$} & 1 & $2378.374^{\mathrm{a}}$ & -0.219 & -0.079 & -0.053 & & $2504.487^{\mathrm{a}}$ & -0.213 & -0.089 & -0.065 & \\
\hline & 2 & & & & & & $2540.161^{\mathrm{a}}$ & -0.214 & -0.094 & -0.071 & \\
\hline & 3 & $2280.081^{\mathrm{a}}$ & -0.237 & -0.083 & -0.058 & & $2572.388^{\mathrm{a}}$ & -0.211 & -0.094 & -0.071 & \\
\hline & 4 & & & & & & $2601.007^{\mathrm{a}}$ & -0.214 & -0.098 & -0.076 & \\
\hline $6-5$ & 18 & & & & & & $1034.144^{\mathrm{b}}$ & -0.289 & 0.145 & 0.185 & QB \\
\hline \multirow[t]{3}{*}{$7-5$} & 18 & $995.415^{\mathrm{b}}$ & -0.463 & 0.207 & 0.253 & QB & & & & & \\
\hline & & & 0.228 & 0.081 & 0.056 & & & 0.213 & 0.094 & 0.071 & \\
\hline & & & 0.326 & 0.137 & 0.153 & & & 0.230 & 0.106 & 0104 & \\
\hline
\end{tabular}

Notes. QB denotes transitions between a quasibound and a bound state. All units are $\mathrm{cm}^{-1}$.

${ }^{a}$ Crofton et al. (1989).

${ }^{\mathrm{b}}$ Carrington et al. (1983).

${ }^{\mathrm{c}}$ rms calculated neglecting QB transitions.

d rms calculated including QB transitions.

choosing the masses. As shown in the present work, it allows for reaching high accuracy in the calculation of the rovibrational transitions. Consequently, benchmark linelists and Einstein A-coefficients have been obtained. The calculations have made use of new very accurate potential energy and DMCs. The results show that the limits for the quality of the PECs and for the adiabatic and non-adiabatic corrections might have been reached, at least for low-lying states. Furthermore, there is no need for any empirical adjustment of the masses so they can expected to be good for general use.

Contrary to $\mathrm{HD}^{+}$and $\mathrm{HD}$, the calculations of Coppola et al., Tung et al., and Engel et al. for $\mathrm{HeH}^{+}$are very accurate (particularly those of Tung et al.). Thus, the improvement of the $\mathrm{HeH}^{+}$results achieved in the present work is clearly due to better vibrational masses. However, to reach the accuracy of $10^{-2} \mathrm{~cm}^{-1}$, the relativistic and QED corrections become relevant. Particularly, the QED effects that increase with increasing $v$ and $J$ (Komasa et al. 2011) become important. This explains the remaining deviations between the present results and the experiment. It should be noted that transition energies involving higher states can be subject to error compensation that needs to be assessed and understood. In any event, the quality of the present results concerning the studied charged and neutral molecular systems is likely state-of-the-art.

J.R.M. acknowledges the CNRS for a one month invited scientist position at the University of Reims Champagne-Ardenne in 2018. Support from the Brazilian agencies CNPq and CAPES is also acknowledged. This work was also supported in part by funds from the Polish National Science Centre granted on the basis of Decision no. DEC-2013/10/E/ST4/00033.

\section{Appendix}

\section{Supplementary Tabular Data Sets}

Twenty nine tables are supplied, containing data for ${ }^{3} \mathrm{HeH}^{+}$, ${ }^{4} \mathrm{HeH}^{+},{ }^{3} \mathrm{HeD}^{+},{ }^{4} \mathrm{HeD}^{+}, \mathrm{HD}^{+}$, and $\mathrm{HD}$ calculated with effective masses. These data are provided in plain format in a tar.gz package.
Table 11

Potential Energy Curve for ${ }^{3} \mathrm{HeH}^{+}$

\begin{tabular}{lc}
\hline \hline $\begin{array}{l}R \\
\text { (a.u.) }\end{array}$ & $\begin{array}{c}\text { Energy } \\
\text { (a.u.) }\end{array}$ \\
\hline 0.3500 & -0.6636438612 \\
0.4000 & -1.2262242865 \\
0.4500 & -1.6362141802 \\
0.5000 & -1.9418274958 \\
0.5500 & -2.1735816512 \\
0.6000 & -2.3516580651 \\
0.6500 & -2.4898769615 \\
0.7000 & -2.5979773598 \\
0.7500 & -2.6829874901 \\
0.8000 & -2.7500815723 \\
\hline
\end{tabular}

Table 12

Dipole Moment Curve for ${ }^{3} \mathrm{HeH}^{+}$

\begin{tabular}{lc}
\hline \hline$R$ & $\begin{array}{c}\text { Dipole } \\
\text { (a.u.) }\end{array}$ \\
\hline 0.3500 & 0.0476670167 \\
0.4000 & 0.0591198908 \\
0.4500 & 0.0719194239 \\
0.5000 & 0.0860850194 \\
0.5500 & 0.1016275588 \\
0.6000 & 0.1185506199 \\
0.6500 & 0.1368513598 \\
0.7000 & 0.1565212256 \\
0.7500 & 0.1775465621 \\
0.8000 & 0.1999092100 \\
\hline
\end{tabular}

Example data for the ${ }^{3} \mathrm{HeH}^{+}$(potential energy and DMCs, rovibrational energy values, $P$-branch data and $R$-branch data) are shown in Tables 11-15; these are from the first 10 lines of data. 
Table 13

Rovibrational Energy Values for ${ }^{3} \mathrm{HeH}^{+}$Computed with Effective Masses

\begin{tabular}{llr}
\hline \hline$v$ & $J$ & $\begin{array}{r}\text { State Energy } \\
\left(\mathrm{cm}^{-1}\right)\end{array}$ \\
\hline 0 & & 0.000000 \\
1 & 0 & 2995.082249 \\
2 & 0 & 5663.172987 \\
3 & 0 & 8002.114235 \\
4 & 0 & 10006.125499 \\
5 & 0 & 11665.761640 \\
6 & 0 & 12968.796813 \\
7 & 0 & 13904.147031 \\
8 & 0 & 14475.464693 \\
9 & 0 & 14736.893395 \\
\hline
\end{tabular}

Table 14

$P$-branch Data for ${ }^{3} \mathrm{HeH}^{+}$Computed with Effective Masses

\begin{tabular}{ccccccc}
\hline \hline$v^{\prime}$ & $J^{\prime}$ & $v^{\prime \prime}$ & $J^{\prime \prime}$ & $\begin{array}{c}A \text {-coefficient } \\
\left(\mathrm{s}^{-1}\right)\end{array}$ & $\begin{array}{c}\text { Upper State Energy } \\
\left(\mathrm{cm}^{-1}\right)\end{array}$ & $\begin{array}{c}\text { Lower State Energy } \\
\left(\mathrm{cm}^{-1}\right)\end{array}$ \\
\hline 1 & 0 & 0 & 1 & 824.763686 & 2995.082249 & 71.369657 \\
1 & 1 & 0 & 2 & 537.166623 & 3060.469917 & 213.668464 \\
$\left(\mathrm{~cm}^{-1}\right)$
\end{tabular}

Table 15

$R$-branch Data for ${ }^{3} \mathrm{HeH}^{+}$Computed with Effective Masses

\begin{tabular}{ccccccc}
\hline \hline$v^{\prime}$ & $J^{\prime}$ & $v^{\prime \prime}$ & $J^{\prime \prime}$ & $\begin{array}{c}A \text {-coefficient } \\
\left(\mathrm{s}^{-1}\right)\end{array}$ & $\begin{array}{c}\text { Upper State Energy } \\
\left(\mathrm{cm}^{-1}\right)\end{array}$ & $\begin{array}{c}\text { Lower State Energy } \\
\left(\mathrm{cm}^{-1}\right)\end{array}$ \\
\hline 0 & 1 & 0 & 0 & 0.106062 & 71.369657 & 0.000000 \\
0 & 2 & 0 & 1 & 1.014328 & 213.668464 & 71.369657 \\
$\left(\mathrm{~cm}^{-1}\right)$
\end{tabular}

\section{ORCID iDs}

Paulo H. R. Amaral (iD https://orcid.org/0000-0003-0799-4143 Leonardo G. Diniz (iD https://orcid.org/0000-0001-6994-2045 Alexander Alijah (iD https://orcid.org/0000-0002-4915-0558 Ludwik Adamowicz (iD https://orcid.org/0000-00019557-0484

José R. Mohallem (iD https://orcid.org/0000-0002-4776-4417

\section{References}

Abgrall, H., Roueff, E., \& Viala, Y. 1982, A\&AS, 50, 505

Alijah, A., \& Duxbury, G. 1990, MolPh, 70, 605

Amaral, P. H. R., \& Mohallem, J. R. 2017, JChPh, 146, 194103

Balint-Kurti, G. G., Moss, R. E., Slader, I. A., \& Shapiro, M. 1990, PhRvA, 41, 4913

Bernath, P., \& Amano, T. 1982, PhRvL, 48, 20
Bethe, H. A., \& Salpeter, E. E. 1977, Quantum Mechanics of One- and Twoelectron Atoms (New York: Plenum)

Bishop, D. M., \& Cheung, L. M 1979, JMoSp, 75, 462

Blom, C. E., Mller, K., \& Filgueira, R. R. 1987, CPL, 140, 489

Bunker, P. R., \& Moss, R. E. 1977, MolPh, 33, 417

Carrington, A., Buttenshaw, J., Kennedy, R. A., \& Softley, T. P. 1981, MolPh, 44, 1233

Carrington, A., Kennedy, R. A., \& Softley, T. P. 1983, CP, 81, 251

Carrington, A., Leach, C. A., Marr, A. J., et al. 1992, CP, 166, 145

Carrington, A., Leach, C. A., Marr, A. J., et al. 1993, JChPh, 98, 5290

Carrington, A., McNab, I. R., \& Montgomerie, C. A. 1989, JPhB, 22, 355

Cencek, W., \& Kutzelnigg, W. 1997, CPL, 266, 383

Chuang, M.-C., \& Zare, R. N. 1987, JMoSp, 121, 380

Coppola, C. M., Lodi, L., \& Tennyson, J. 2011, MNRAS, 415, 487

Coxon, J. A., \& Hajigeorgiou, P. G. 1999, JMoSp, 193, 306

Crofton, M. W., Altman, R. S., Haese, N. N., \& Oka, T. 1989, JChPh, 91, 5882

Diniz, L. G., Alijah, A., Adamowicz, L., \& Mohallem, J. R. 2015, CPL, 633, 89

Diniz, L. G., Alijah, A., \& Mohallem, J. R. 2012, JChPh, 137, 164316 
Diniz, L. G., Alijah, A., \& Mohallem, J. R. 2018, ApJS, 235, 35

Diniz, L. G., Kirnosov, N., Alijah, A., Mohallem, J. R., \& Adamowicz, L. 2016, JMoSp, 322, 22

Engel, E. A., Doss, N., Harris, G. J., \& Tennyson, J. 2005, MNRAS, 357, 471

Esry, B. D., \& Sadeghpour, H. R. 1999, PhRvA, 60, 3604

Galli, D., \& Palla, F. 1998, A\&A, 335, 403

Gonçalves, C., \& Mohallem, J. R. 2004, JCoCh, 25, 1736

Güsten, R., Wiesemeyer, H., Neufeld, D., et al. 2019, Natur, 568, 357

Hirshfeld, F. L. 1977, AcTC, 44, 129

Hoyland, J. R. 1967, JChPh, 47, 49

Ishikawaa, A., Nakashima, H., \& Nakatsuji, H. 2012, CP, 401, 62

Jiang, S., Yu, C., Yuan, G., Wu, T., \& Lu, R. 2017, NatSR, 7, 42086

Jones, K., Formanek, M., Mazumder, R., Kirnosov, N., \& Adamowicz, L. 2016a, MolPh, 114, 1634

Jones, K., Kirnosov, N., Sharkey, K. L., \& Adamowicz, L. 2016b, MolPh, 114, 2052

Kediziera, D., Stanke, M., Bubin, S., Barysz, M., \& Adamowicz, L. 2006 JChPh, 15, 014318

Kolos, W., \& Peek, J. M. 1976, CP, 12, 381

Komasa, J., Piszczatowski, K., Ach, G., et al. 2011, J. Chem. Theory Comput., 7, 3105

Korobov, V. I. 2006, PhRvA, 74, 052506

Korobov, V. I. 2008, PhRvA, 77, 022509

Kutzelnigg, W. 2007, MolPh, 105, 2627

Lepp, S., Stancil, P. C., \& Dalgarno, A. 2002, JPhB, 35, R57

LeRoy, R. J. 2017, JQSRT, 186, 167

Lillestonen, T. C., \& Wheatley, R. J. 2008, ChCom, 45, 5909

Liu, D.-J., Ho, W.-C., \& Oka, T. 1987, JChPh, 87, 2442

Liu, Z., \& Davies, P. B. 1997a, JChPh, 107, 337

Liu, Z., \& Davies, P. B. 1997b, PhRvL, 79, 2779
Matsushima, F., Oka, T., \& Tagaki, K. 1997, PhRvL, 78, 1664

Mohallem, J. R., Diniz, L. G., \& Dutra, A. S. 2011, CPL, 501, 575

Nakashima, H., Hijikata, Y., \& Nakatsuji, H. 2013, ApJ, 770, 144

Nakashima, H., \& Nakatsuji, H. 2013, JChPh, 139, 074105

Pachucki, K. 2012, PhRvA, 85, 042511

Pachucki, K., \& Komasa, J. 2008, PhRvA, 78, 052503

Pachucki, K., \& Komasa, J. 2009, JChPh, 130, 164113

Pachucki, K., \& Komasa, J. 2010, PCCP, 12, 9188

Pachucki, K., \& Komasa, J. 2012, JChPh, 137, 204314

Patra, S., Karr, J.-P., Hilico, L., et al. 2018, JPhB, 51, 024003

Pavanello, M., Cafiero, M., Bubin, S., \& Adamowicz, L. 2008, IJQC, 108, 2291

Perry, A. J., Hodqesl, J. N., Markusl, C. R., Kocherill, G. S., \& McCall, B. J. J. 2014, JChPh, 141, 101101

Purder, J., Civis, S., Blom, C. E., \& van Hemert, M. C. 1992, JMoSp, 153, 701

Roberge, W., \& Dalgarno, A. 1982, ApJ, 255, 489

Rothman, L. S., Jacquemart, D., Barbe, A., et al. 2017, JQSRT, 203, 3

Schmidt, M. W., Baldridge, K. K., Boatz, J. A., et al. 1993, JCoCh, 14, 1347

Shen, J., Borodin, A., Hansen, M., \& Schiller, S. 2012, PhRvA, 85, 032519

Sprecher, D., Liu, J., Jungen, C., Ubachs, W., \& Merkt, F. 2010, JChPh, 133, 111102

Stanke, M., Palikot, E., \& Adamowicz, L. 2016a, JChPh, 144, 174101

Stanke, M., Palikot, E., Kediziera, D., \& Adamowicz, L. 2016b, JChPh, 144, 224111

Tennyson, J., Yurchenko, S. N., Al-Refaie, A. F., et al. 2016, JMoSp, 327, 73

Tolliver, D. E., Kyrala, G. A., \& Wing, W. H. 1979, PhRvL, 43, 1719

Tung, W.-C., Pavanello, M., \& Adamowicz, L. 2012, JChPh, 137, 164305

Wolniewicz, L. 1995, JChPh, 103, 1792

Wolniewicz, L., \& Poll, J. D. 1986, MolPh, 59, 953 